\title{
A NEW METHOD OF ANGULAR CORRELATION MEASUREMENTS*
}

\author{
R. R. LEWIS, Jr. \\ Department of Physics, University of Michigan, Ann Arbor, Michigan, U.S.A.
}

Received 17 March 1969

A new type of angular correlation apparatus is described, and a mathematical model of its statistics is given. The angular correlation of coincident nuclear radiations can be measured by detecting intensity correlations in the output of two counters. For prompt coincidences, the detector currents are mixed in a broadband circuit whose output is the product of the two inputs. The time-averaged output of the mixer is shown to be proportional to the rate of true coincidences, and therefore to the angular correla-

\section{Introduction}

Pulse counting has always been the cornerstone for detection of nuclear radiations. Nevertheless, it can be advantageous to operate counters with intense sources, so that their output is a continuous fluctuating current rather than a sequence of resolved pulses. Some recent symmetry experiments ${ }^{1}$ ), which stimulated this work, have used counters as rate meters for precision measurement of singles rates. We will show that it also is possible to use continuous currents for angular correlation experiments normally done by counting pulse coincidences. Contrary to one's intuition, the rate of true coincidences can still be determined even when the individual pulses overlap; moreover, the statistical errors are the same.

The subject will be presented as alternative limits of one general problem: detection of current correlations, for weak or strong sources. The purpose of this work is to devise a unified treatment of random currents, valid in both extremes, and to apply it to a systematic derivation of statistical errors. The main result is a proof of the equivalence of pulsed and continuous currents. There is a correspondence between circuit elements: instead of a scaler one uses a meter; instead of a pulse shaping circuit, a filter; instead of a coincidence circuit, a mixer; etc. And there is a correspondence of the outputs and of their fluctuations. The central point is that the statistical behavior of pulsed currents is unchanged when the pulses overlap, so long as linearity of the circuit is maintained.

We conclude that the use of strong sources and continuous currents offers a feasible alternative to conventional coincidence experiments. One can measure in this way angular distributions, lifetimes, magnetic moments, quadrupole splittings. Although the

* Research sponsored by Air Force Office of Scientific Research, under grant no. 1288-67. tion function. Furthermore, the fluctuation of this output has the same ratio to the average current as the rate of random to true coincidences both for weak and strong sources. For delayed coincidences, other circuits give the time spectrum and the frequency spectrum of the perturbed correlation function. Possible applications are mentioned, and the relation to the Brown-Twiss interferometer is clarified.

original motivation was to make possible precision measurements of perturbed angular correlations for symmetry tests ${ }^{2}$ ), the techniques are of broader utility. One advantage will be the direct measurement of the frequency spectrum (rather than the time spectrum) of delayed coincidences, especially for long-lived states. The observation of sharp peaks due to individual intermediate states, will offset the loss of energy resolution available in pulse technology.

\section{Elementary discussion}

Before we consider a mathematical description, it is worthwhile discussing the basic ideas in their simplest form. The heuristic arguments used here will be borne out by derivations in the next section.

\subsection{Single Rates}

Consider first the output of a single counter as the source strength increases. For a weak source, it consists of random current pulses with shape $F(t)$. Of course the reason for the appearance of pulses is the quantum nature of the radiation, but our description of the final pulses will be classical. For simplicity we take identical rectangular pulses described by two parameters, the width $\Delta t$ and the integrated charge $q=\int \mathrm{d} t F(t)$. For a source with $v$ decays per second the mean pulse rate is $v \varepsilon$, where $\varepsilon$ is a small factor including both solid angle and efficiency of the counter. If $v \varepsilon \Delta t \ll 1$, the pulses are resolved and the rate can be determined with a scaler. The mean number of counts in time $T$ is

$$
\langle N\rangle=v \varepsilon T,
$$

and the uncertainty in this number can be predicted if the counts follow Poisson statistics. The fluctuation $\Delta N=\sqrt{ }\left(\left\langle N^{2}\right\rangle-\langle N\rangle^{2}\right)$ is then $\sqrt{ }\langle N\rangle$ and so the relative error is 


$$
\Delta N /\langle N\rangle=1 / \sqrt{ }(v \varepsilon T) .
$$

For stronger sources $(v \varepsilon \Delta t \gtrsim 1)$ we can use a meter in place of the scaler; the mean current* is

$$
\langle I\rangle=v \varepsilon q \text {. }
$$

So long as the circuit remains linear, the mean current can be calibrated and used as a measure of the singles rate. There will be an uncertainty in this measurement due to fluctuations in the current $I(t)$, which we can estimate if we can predict the autocorrelation of the current $\langle I(t) I(t+\tau)\rangle$. This function tells us both the mean square current (put $\tau \rightarrow 0$ ) and the frequency spectrum of the power [compute the Fourier transform $\left.\int \mathrm{d} \tau \exp (\mathrm{i} \omega t)\langle I(t) I(t+\tau)\rangle\right]$.

We will analyze the autocorrelation by considering the variations from the mean current $\langle I(t)\rangle$. Writing $I(t)=\langle I\rangle+i(t)$, we find

$$
\langle I(t) I(t+\tau)\rangle=\langle I\rangle^{2}+\langle i(t) i(t+\tau)\rangle .
$$

These two terms have a simple significance in terms of the underlying pulses which comprise $I(t)$ : the first term is due to products of two different pulses, and the second term is due to the product of each pulse with itself. The first term is

$$
\langle I\rangle^{2}=v^{2} \varepsilon^{2} q^{2},
$$

which grows like the number of different pulses and thus like $v^{2}$. It is independent of $\tau$ and only contributes to the dc power. The reason for this is that different pulses have a random distribution of arrival times, and if one set of pulses is delayed the distribution remains random. Hence the average product is the same for any delay time $\tau$. If we consider each pulse as a superposition of sine waves, then two different pulses have sinusoidal components with different phases. The random distribution of arrival times implies a random phase difference and all the ac components average to zero.

The second term is obtained by multiplying each pulse with itself and summing

$$
\langle i(t) i(t+\tau)\rangle=v \varepsilon \int \mathrm{d} t_{1} F\left(t_{1}\right) F\left(t_{1}+\tau\right) .
$$

The auto-correlation grows like $v$, but persists only over the width of the individual pulses and is shown in fig. 1 . The corresponding power spectrum extends over

\footnotetext{
* Throughout this section the mean value will be taken as the time average over a long time interval $\langle f(t)\rangle=(1 / T) \int_{0}^{T} \mathrm{~d} t \mathrm{f}(t)$. We will always deal with "stationary" quantities with mean values $\langle f(t)\rangle$ independent of time: there is no preferred time origin for our averages.
}

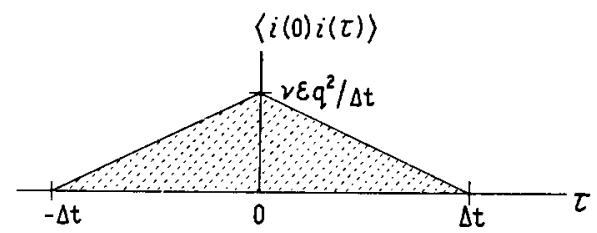

Fig. 1. The auto-correlation $\langle i(0) / i(\tau)\rangle$ vs time delay is shown for rectangular pulses.

the entire frequency spectrum of the pulse, $0 \leqq \omega \leqq 1 / \Delta t$. The mean square deviation from the average current is

$$
\left\langle i^{2}(t)\right\rangle=v \varepsilon q^{2} / \Delta t .
$$

Now we can estimate the errors in measuring the singles rate with a meter. The average reading is given by eq. (3), and the fluctuation $\Delta I=\sqrt{ }\left(\left\langle I^{2}\right\rangle-\langle I\rangle^{2}\right)=$ $\sqrt{ }\left(\left\langle i^{2}\right\rangle\right)$ by eq. (7), so the relative error is

$$
\Delta I /\langle I\rangle=1 / \sqrt{ }(v \varepsilon \Delta t) \text {. }
$$

Comparison with eq. (2) shows that this is just the error in counting the number of pulses arriving in time $\Delta t$. This is a relatively large error, since all the frequencies in the spectrum of $i$ contribute.

The error can be reduced by integrating the current over a long time, and measuring the total charge $Q=\int_{0}^{T} \mathrm{~d} t I(t)$. The mean charge is

$$
\langle Q\rangle=\int_{0}^{T} \mathrm{~d} t\langle I(t)\rangle=v \varepsilon q T,
$$

and the charge fluctuation is

$$
(\Delta Q)^{2}=\left\langle Q^{2}\right\rangle-\langle Q\rangle^{2}=\int_{0}^{T} \mathrm{~d} t_{1} \int_{0}^{T} \mathrm{~d} t_{2}\left\langle i\left(t_{1}\right) i\left(t_{2}\right)\right\rangle .
$$

But we have seen that the auto-correlation only extends over short time differences

$$
\left\langle i\left(t_{1}\right) i\left(t_{2}\right)\right\rangle \simeq \begin{cases}\left\langle i^{2}\right\rangle & \text { if } t_{1}-t_{2} \lesssim \Delta t, \\ 0 & \text { if } \Delta t \lesssim t_{1}-t_{2},\end{cases}
$$

and so using eq. (7) and eq. (10) to evaluate the integral, we find

$$
\Delta Q=\sqrt{ }\left(v \varepsilon q^{2} T\right),
$$

and the relative error in this measurement is

$$
\Delta Q /\langle Q\rangle=1 / \sqrt{ }(v \varepsilon T),
$$

which is identical with eq. (2). An alternative to measuring the integrated current is to insert a low frequency filter before the meter; again one finds eq. (2) with $T$ replaced by the reciprocal of the bandwidth. This example has demonstrated the equivalence of pulse 
and current measurements, at least for finding singles rates.

\subsection{COINCIDENCE RATES}

Next we want to demonstrate that this equivalence also works for coincidence rates. Consider two counters, $a$ and $b$, exposed to a source with two radiations in prompt coincidence. For clarity we will let counter a respond only to one radiation, and counter $b$ to the other. For a weak source, the rate of true coincidences is $v \varepsilon^{2} W$ and the rate of chance coincidences is $2 v^{2} \varepsilon^{2} \Delta t$, with ratio $W / 2 v \Delta t$. Here $W$ is the angular correlation function and $\varepsilon$ is the solid angle. In counting time $T$, the mean number of true coincidences is

$$
\left\langle N_{\text {true }}\right\rangle=v \varepsilon^{2} W T \text {. }
$$

The fluctuation about this mean will be the sum of the fluctuations in both true and chance coincidences,

$$
\Delta N=\sqrt{ }\left(\Delta N_{\text {true }}^{2}+\Delta N_{\text {chance }}^{2}\right) \text {. }
$$

Assuming Poisson statistics for both numbers, $\Delta N=$ $\sqrt{ }\left(\left\langle N_{\text {true }}\right\rangle+\left\langle N_{\text {chance }}\right\rangle\right)$ and the relative error in measuring the true rate is

$$
\begin{aligned}
\Delta N /\left\langle N_{\text {true }}\right\rangle & =\sqrt{ }\left(\left\langle N_{\text {true }}\right\rangle+\left\langle N_{\text {chance }}\right\rangle\right) /\left\langle N_{\text {true }}\right\rangle= \\
& =\sqrt{ }\left\{(1+2 v \Delta t / W) / v \varepsilon^{2} W T\right\} .
\end{aligned}
$$

As $v$ increases, this error approaches a constant

$$
\Delta N /\left\langle N_{\text {true }}\right\rangle \cong(1 / \varepsilon W) \sqrt{ }(2 \Delta t / T),
$$

determined by the circuit parameters, but independent of the source strength. The "signal" $\left\langle N_{\text {true }}\right\rangle$ increases linearly with $v$, but so does the "noise" $\Delta N_{\text {chance }}=$ $\sqrt{ }\left\langle N_{\text {chance }}\right\rangle$. These are the formulas customarily used in analyzing the statistics of coincidence circuits.

These same results govern the performance of the new coincidence circuit shown in fig. 2 . The current from each detector $\left(I_{\mathrm{a}}\right.$ and $\left.I_{\mathrm{b}}\right)$ passes through a filter and into a mixer whose output $\left(I_{\mathrm{c}}\right)$ is proportional to the product of the two inputs. The output is then integrated over a long time constant, or passed through a narrow-band filter to a meter. The entire circuit up to the mixer must pass frequencies up to $\simeq 1 / \Delta t$ and have time jitter less than $\Delta t$. An angular correlation

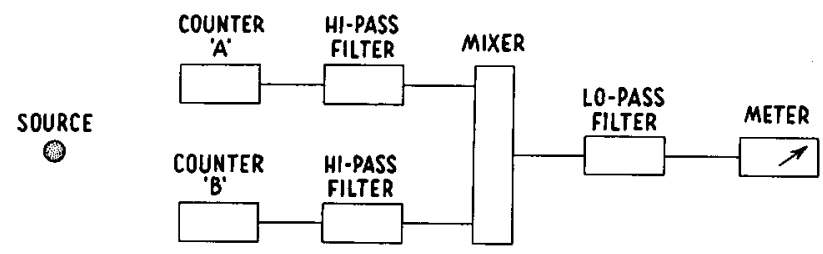

Fig. 2. Block diagram of new angular correlation apparatus for measuring rate of true prompt coincidences. experiment can be performed by measuring the dc output of the mixer versus the angle between the two counters.

The trick in understanding this circuit is to recognize the similarity between the mean current output of the mixer and the fluctuation in the current from a single counter. In this case we must evaluate the crosscorrelation of two currents $\left\langle I_{\mathrm{a}}(t) I_{\mathrm{b}}(t)\right\rangle$ rather than the auto-correlation of a single current $\langle I(t) I(t+\tau)\rangle$. By the same reasoning, the cross-correlation can be split into two terms by introducing the deviations from the mean $I_{\mathrm{a}}=\left\langle I_{\mathrm{a}}\right\rangle+i_{\mathrm{a}}, I_{\mathrm{b}}=\left\langle I_{\mathrm{b}}\right\rangle+i_{\mathrm{b}}$ :

$$
\left\langle I_{\mathrm{c}}(t)\right\rangle=\left\langle I_{\mathrm{a}}(t) I_{\mathrm{b}}(t)\right\rangle=\left\langle I_{\mathrm{a}}\right\rangle \cdot\left\langle I_{\mathrm{b}}\right\rangle+\left\langle i_{\mathrm{a}}(t) i_{\mathrm{b}}(t)\right\rangle .
$$

The first term results from chance coincidences in the two counters and grows like $v^{2}$

$$
\left\langle I_{\mathrm{a}}\right\rangle\left\langle I_{\mathrm{b}}\right\rangle=v^{2} \varepsilon^{2} q^{2} .
$$

The second term comes from true coincidences and is proportional to $v$ and to the angular correlation function,

$$
\left\langle i_{\mathrm{a}}(t) i_{\mathrm{b}}(t)\right\rangle=v \varepsilon^{2} q^{2} W / \Delta t .
$$

Both terms represent dc currents in the mixer output.

But it is evident that the two terms can be measured separately: any linear device which cancels the $\mathrm{dc}$ current in the output of the counters will eliminate the chance coincidences in the dc output of the mixer. A high-pass filter with broad bandwidth will suffice; so will a battery.

We will not attempt to give an elementary discussion of the fluctuations in the mixer output, which would require the estimation of biquadratic averages such as $\left\langle I_{\mathrm{a}}^{2} I_{\mathrm{b}}^{2}\right\rangle$. Some qualitative results are clear, however. Since our "signal" is again the dc output, the errors in its determination can be reduced by integrating out (or filtering) the high frequency components. The integration time (or reciprocal bandwidth) will play the role of the counting time in a pulse circuit. The statistical error will be shown in the next section to be the same as eq. (15),

$$
\Delta I_{\mathrm{c}} /\left\langle I_{\mathrm{c}}\right\rangle=\sqrt{ }\left\{(1+2 v R / W) / v \varepsilon^{2} W T\right\},
$$

with a suitable interpretation of the resolving time $R$ and counting time $T$. The circuit in fig. 2 will work for any source strength, weak or strong, provided the linearity is maintained. The result stated in eq. (20) shows that the statistical error approaches a constant as the pulses overlap. The signal to noise ratio in the output is then determined by the solid angle $\varepsilon$, the rise time of the pulses $R$ and the time constant governing circuit stability $T$. It remains to be seen how large this signal to noise ratio can be made. 
This shows another example of the equivalence of pulses and currents. We conclude that there is a very general relationship between the two, reminiscent of the particle-wave duality in quantum mechanics.

\section{Statistical model of random detector currents}

In order to analyze circuits like fig. 2 , we need a model for random detector currents. It is not sufficient merely to characterize the currents as - say-Gaussian noise plus a signal. We want to predict the signal to noise ratio, and study its dependence on circuit parameters. The treatment we have adopted is a modification of the theory of shot noise in electronic devices ${ }^{3,4}$ ). The reader is urged to consult the articles of Rice $^{3}$ ) for a discussion of the basic statistical concepts. The essential step is to isolate the random variables $(r)$ from the non-random variables $(n)$. The output of any circuit $I(n, r)$ must be calculated for any configuration of variables $n$ and $r$, and then averaged over the probability $p(r)$. In treating the various observable effects of random currents, it is vital to perform the average over $r$ for the final output, and not at some intermediate stage. The general form of the observables is

$$
\begin{aligned}
\langle I(n)\rangle & =\int \mathrm{d} r p(r) I(n, r), \\
\left\langle I\left(n_{1}\right) I\left(n_{2}\right)\right\rangle & =\int \mathrm{d} r p(r) I\left(n_{1} r\right) I\left(n_{2} r\right),
\end{aligned}
$$

and so forth. For linear circuits we usually need only a few such averaged quantities.

\subsection{Singles PULSE RATES}

For a random current composed of pulses, the use of conditional probabilities is appropriate: one considers the probability $p(K)$ of exactly $K$ pulses in time $T$, and defines the conditional probability $p(r \mid K)$ of the configuration $r$ for $K$ pulses. The general form of an averaged quantity is changed to

$$
\langle I(n)\rangle=\sum_{K=0}^{\infty} p(K) \int \mathrm{d} r p(r \mid K) I_{K}(n, r),
$$

where $I_{K}$ is the current produced by $K$ pulses. The probability $p(K)$ can always be taken, for weak or strong sources, as the Poisson distribution

$$
p(K)=\left(\langle N\rangle^{K} / K !\right) \exp (-\langle N\rangle),
$$

where $\langle N\rangle$ is the mean number of pulses in time $T$.

To describe the current from a single detector, we can take as random variables the arrival times $t_{j}$ of the individual pulses; the time $t$ is the only non-random variable. If each $t_{j}$ is independently and uniformly distributed over the time $T$, the conditional probability is

$$
p\left(t_{j} \mid K\right)=(1 / T)^{K} .
$$

The current is just the sum of $K$ pulses

$$
I_{K}\left(t, t_{j}\right)=\sum_{j=1}^{K} F\left(t-t_{j}\right) .
$$

These eqs. (22)-(25) define our model; all that remains is straightforward calculation. The mean current is

$$
\begin{aligned}
\langle I(t)\rangle=\sum_{K} p(K) \int_{-T / 2}^{T / 2}(1 / T) \mathrm{d} t_{1} \ldots \\
\ldots \int_{-T / 2}^{T / 2}(1 / T) \mathrm{d} t_{K} \sum_{j} F\left(t-t_{j}\right) .
\end{aligned}
$$

The sum has $K$ identical terms

$$
\langle I(t)\rangle=\sum_{K} K p(K)(1 / T) \int \mathrm{d} t_{1} F\left(t-t_{1}\right),
$$

using $\sum K p(K)=\langle N\rangle=v \varepsilon T$, and shifting time variables we get

$$
\langle I(t)\rangle=v \varepsilon \int F\left(t_{1}\right) \mathrm{d} t_{1} .
$$

Throughout these derivations we will assume that $T$ is much longer than the pulse width, and discard contributions of pulses overlapping the ends of the time interval. The results describe "stationary random currents", which are independent of the time origin. But we will not actually take the limit $T \rightarrow \infty$, since in any real experiment the counting time $T$ is limited by long term drifts in the circuit parameters.

The autocorrelation of the current is

$$
\begin{aligned}
\langle I(t) I(t+\tau)\rangle= & \sum_{K} p(K) \int(1 / T) \mathrm{d} t_{1} \ldots \\
& \int(1 / T) \mathrm{d} t_{K} \sum_{i} F\left(t-t_{i}\right) \sum_{j} F\left(t-t_{j}+\tau\right) .
\end{aligned}
$$

Splitting the double sum into $K(K-1)$ identical offdiagonal terms and $K$ identical diagonal terms, we get

$$
\begin{aligned}
& \langle I(t) I(t+\tau)\rangle= \\
& =\sum K(K-1) p(K)\left(1 / T^{2}\right) \\
& \quad \cdot \int \mathrm{d} t_{1} F\left(t-t_{1}\right) \int \mathrm{d} t_{2} F\left(t-t_{2}+\tau\right)+ \\
& \quad+\sum K p(K)(1 / T) \int \mathrm{d} t_{1} F\left(t-t_{1}\right) F\left(t-t_{1}+\tau\right) .
\end{aligned}
$$

Changing integration variables and using

$$
\sum K(K-1) p(K)=(v \varepsilon T)^{2}
$$

leads to the result 


$$
\begin{aligned}
& \langle I(t) I(t+\tau)\rangle= \\
& \quad=\left[v \varepsilon \int \mathrm{d} t_{1} F\left(t_{1}\right)\right]^{2}+v \varepsilon \int \mathrm{d} t_{1} F\left(t_{1}\right) F\left(t_{1}+\tau\right) .
\end{aligned}
$$

From this we find the mean square current fluctuation

$$
(\Delta I)^{2}=\left\langle I^{2}(t)\right\rangle-\langle I(t)\rangle^{2}=v \varepsilon \int F^{2}\left(t_{1}\right) \mathrm{d} t_{1} .
$$

These results confirm our previous arguments, and extend the formulas to pulses of arbitrary shape. The process could be continued to higher ordered correlations like $\left\langle I^{4}\right\rangle$ but we will not do so. For strong sources, the central limit theorem guarantees that the random current is a Gaussian process, and that these higher correlations are determined by the lower ones; for example, if $v \varepsilon \Delta t \gg 1$, then $\left\langle I^{4}(t)\right\rangle \cong 3\left\langle I^{2}(t)\right\rangle^{2}$. In this sense, equations $\left(3^{\prime}\right)$ and $\left(6^{\prime}\right)$ provide a complete characterization of the current for strong sources.

So far, our treatment is identical with the theory of shot noise. It is important to recognize that another choice of the random variables will give the same observable results; it consists of attributing random variables to the source, instead of to the counter. Let there be $K$ quanta emitted by the source, at times $t_{j}$ and in directions $\hat{n}_{j}$. If the times and directions are independently and uniformly distributed, the conditional probability is

$$
p\left(t_{j}, \hat{n}_{j} \mid K\right)=(4 \pi T)^{-K} .
$$

The non-random variables are the time $t$ and the direction of the counter $\hat{n}$. The current can be written

$$
I_{K}\left(t \hat{n} ; t_{j}, \hat{n}_{j}\right)=\sum_{j=1}^{K} S\left(\hat{n}, \hat{n}_{j}\right) F\left(t-t_{j}\right),
$$

where $S$ is a step function which is unity if $\hat{n}_{j}$ is within the solid angle subtended by the counter at $\hat{n}$, and $S$ is zero otherwise. It is an easy exercise to repeat the derivations; the results are identical, if we take $p(K)$ with mean $\langle N\rangle=v T$ and use $(1 / 4 \pi) \int \mathrm{d} \Omega_{1} S\left(\hat{n}, \hat{n}_{1}\right)=\varepsilon$. This is another model of a random current; there may be many more. The device of relating the random variables to the source is crucial to our next problem: the study of cross-correlations in two counters looking at the same source.

\subsection{COINCIDENCE RATES}

As before, we will consider a source emitting two different radiations ( $a$ and $b$ ) in prompt coincidence. The times $t_{j}$ and directions $\hat{n}_{\mathbf{j}}^{\mathrm{a}}$ and $\hat{n}_{j}^{\mathrm{b}}$ of the two quanta are random variables, while the time $t$ and directions of the two counters $\hat{n}^{\mathrm{a}}$ and $\hat{n}^{\mathrm{b}}$ are nonrandom variables.
The times are distributed as before, but the relative directions of the two quanta from the same decay are correlated. The conditional probability is

$$
\begin{aligned}
p\left(t_{1} \hat{n}_{1}^{\mathrm{a}} n_{1}^{\mathrm{b}}, t_{2} \hat{n}_{2}^{\mathrm{a}} \hat{n}_{2}^{\mathrm{b}}, . . \mid K\right)= \\
=(1 / T)^{K} p\left(\hat{n}_{1}^{\mathrm{a}} \hat{n}_{1}^{\mathrm{b}}\right) p\left(\hat{n}_{2}^{\mathrm{a}} \hat{n}_{2}^{\mathrm{b}}\right) \ldots p\left(\hat{n}_{K}^{\mathrm{a}} \hat{n}_{K}^{\mathrm{b}}\right),
\end{aligned}
$$

and the detector currents are

$$
\begin{gathered}
I_{\mathrm{a}}=\sum_{j} S\left(\hat{n}^{\mathrm{a}}, \hat{n}_{j}^{\mathrm{a}}\right) F_{\mathrm{a}}\left(t-t_{j}\right), \\
I_{\mathrm{b}}=\sum_{j} S\left(\hat{n}^{\mathrm{b}}, \hat{n}_{j}^{\mathrm{b}}\right) F_{\mathrm{b}}\left(t-t_{j}\right) .
\end{gathered}
$$

We are including some difference in the pulse shapes in the two counters, but are neglecting the current in counter a due to quanta $b$ and vice versa. The probability $p\left(\hat{n}_{1}^{\mathrm{a}} \hat{n}_{1}^{\mathrm{b}}\right)$ is normalized so that

$$
\int \mathrm{d} \Omega_{1}^{\mathrm{a}} \int \mathrm{d} \Omega_{1}^{\mathrm{b}} p\left(\hat{n}_{1}^{\mathrm{a}}, \hat{n}_{1}^{\mathrm{b}}\right)=1 .
$$

It is easily shown that the mean current and the auto-correlation in either circuit is exactly as above. But there is also a cross-correlation in the two currents,

$$
\begin{aligned}
& \left\langle I_{\mathrm{a}}(t) I_{\mathrm{b}}(t+\tau)\right\rangle= \\
& \sum_{K} p(K) \int(1 / T) \mathrm{d} t_{1} \int \mathrm{d} \Omega_{1}^{\mathrm{a}} \int \mathrm{d} \Omega_{1}^{\mathrm{b}} p\left(\hat{n}_{1}^{\mathrm{a}} \hat{n}_{1}^{\mathrm{b}}\right) \ldots \\
& \quad \ldots \int(1 / T) \mathrm{d} t_{K} \int \mathrm{d} \Omega_{K}^{\mathrm{a}} \int \mathrm{d} \Omega_{K}^{\mathrm{b}} p\left(\hat{n}_{K}^{\mathrm{a}} \hat{n}_{K}^{\mathrm{b}}\right) . \\
& \cdot \sum_{i} S\left(\hat{n}^{\mathrm{a}}, \hat{n}_{i}^{\mathrm{a}}\right) F_{\mathrm{a}}\left(t-t_{i}\right) \sum_{j} S\left(\hat{n}^{\mathrm{b}}, \hat{n}_{j}^{\mathrm{b}}\right) F_{\mathrm{b}}\left(t+\tau-t_{j}\right) .
\end{aligned}
$$

Again we separate the $K(K-1)$ off-diagonal terms (chance coincidences) from the $K$ diagonal terms (true coincidences). The latter are proportional to the double integral

$$
\varepsilon^{2} W\left(\hat{n}^{\mathrm{a}}, \hat{n}^{\mathrm{b}}\right) \equiv \int \mathrm{d} \Omega_{1}^{\mathrm{a}} \int \mathrm{d} \Omega_{1}^{\mathrm{b}} p\left(\hat{n}_{1}^{\mathrm{a}} \hat{n}_{1}^{\mathrm{b}}\right) S\left(\hat{n}^{\mathrm{a}}, \hat{n}_{1}^{\mathrm{a}}\right) S\left(\hat{n}^{\mathrm{b}}, \hat{n}_{1}^{\mathrm{b}}\right),
$$

which defines the correlation function $W$. It is normalized so that

$$
\int \mathrm{d} \Omega^{\mathrm{a}} \int \mathrm{d} \Omega^{\mathrm{b}} W\left(\hat{n}^{\mathrm{a}}, \hat{n}^{\mathrm{b}}\right)=1 .
$$

The quantity $\varepsilon^{2}$ is the product of the two solid angles $\varepsilon^{a} \varepsilon^{b}$. Evaluation of eq. (31) gives

$$
\begin{aligned}
\left\langle I_{\mathrm{a}}(t) I_{\mathrm{b}}(t+\tau)\right\rangle=v^{2} \varepsilon^{2} \int \mathrm{d} t_{1} F_{\mathrm{a}}\left(t_{1}\right) \int \mathrm{d} t_{2} F_{\mathrm{b}}\left(t_{2}\right)+ & \\
& +v \varepsilon^{2} W \int \mathrm{d} t_{1} F_{\mathrm{a}}\left(t_{1}\right) F_{\mathrm{b}}\left(t_{1}+\tau\right) .
\end{aligned}
$$

The first term is obviously the product of the two mean 
currents and can be removed by introducing the ac currents,

$$
\begin{array}{r}
\left\langle i_{\mathrm{a}}(t) i_{\mathrm{b}}(t+\tau)\right\rangle \equiv\left\langle\left[I_{\mathrm{a}}(t)-\left\langle I_{\mathrm{a}}\right\rangle\right]\left[I_{\mathrm{b}}(t+\tau)-\left\langle I_{\mathrm{b}}\right\rangle\right]\right\rangle= \\
=v \varepsilon^{2} W \int \mathrm{d} t_{1} F_{\mathrm{a}}\left(t_{1}\right) F_{\mathrm{b}}\left(t_{1}+\tau\right) .
\end{array}
$$

Another simple trick for eliminating the first term, is to insert a linear circuit whose output pulses $G(t)$ satisfy

$$
\int \mathrm{d} t_{1} G\left(t_{1}\right)=0
$$

This establishes our principal result; the mean output of the mixer in fig. 2 is

$$
\left\langle I_{\mathrm{c}}(t)\right\rangle=\left\langle i_{\mathrm{a}}(t) i_{\mathrm{b}}(t)\right\rangle=v \varepsilon^{2} W \int \mathrm{d} t_{1} G^{\mathrm{a}}\left(t_{1}\right) G^{\mathrm{b}}\left(t_{1}\right),
$$

proportional to the correlation function $W$.

The evaluation of the fluctuation in the mixer output requires the auto-correlation in $I_{\mathrm{c}}(t)$. The model gives a straightforward technique for deriving this,

$$
\begin{aligned}
& \left\langle I_{\mathrm{c}}(t) I_{\mathrm{c}}(t+\tau)\right\rangle= \\
& =\sum_{K} p(K) \int(1 / T) \mathrm{d} t_{1} \int \mathrm{d} \Omega_{1}^{\mathrm{a}} \int \mathrm{d} \Omega_{1}^{\mathrm{b}} p\left(\hat{n}_{1}^{\mathrm{a}} \hat{n}_{1}^{\mathrm{b}}\right) \ldots \\
& \quad \ldots \int(1 / T) \mathrm{d} t_{K} \int \mathrm{d} \Omega_{K}^{\mathrm{a}} \int \mathrm{d} \Omega_{K}^{\mathrm{b}} p\left(\hat{n}_{K}^{\mathrm{a}} \hat{n}_{K}^{\mathrm{b}}\right) \sum_{i} S_{i}^{\mathrm{a}} G_{\mathrm{a}}\left(t-t_{i}\right) . \\
& \quad \cdot \sum_{j} S_{j}^{\mathrm{b}} G_{\mathrm{b}}\left(t-t_{j}\right) \sum_{k} S_{k}^{\mathrm{a}} G_{\mathrm{a}}\left(t+\tau-t_{k}\right) \sum_{l} S_{l}^{\mathrm{b}} G_{\mathrm{b}}\left(t+\tau-t_{l}\right),
\end{aligned}
$$

which has $K^{4}$ terms. However, from eq. (36) we need keep only the terms with indices equal in pairs. A short derivation gives

$$
\begin{aligned}
& \left\langle I_{\mathrm{c}}(t) I_{\mathrm{c}}(t+\tau)\right\rangle= \\
& \quad\left[v \varepsilon^{2} W \int \mathrm{d} t_{1} G_{\mathrm{a}}\left(t_{1}\right) G_{\mathrm{b}}\left(t_{1}\right)\right]^{2}+ \\
& \quad+v^{2} \varepsilon^{4} W^{2} \int \mathrm{d} t_{1} G_{\mathrm{a}}\left(t_{1}\right) G_{\mathrm{b}}\left(t_{1}+\tau\right) \int \mathrm{d} t_{2} G_{\mathrm{b}}\left(t_{2}\right) G_{\mathrm{a}}\left(t_{2}+\tau\right)+ \\
& \quad+v \varepsilon^{2} W \int \mathrm{d} t_{1} G_{\mathrm{a}}\left(t_{1}\right) G_{\mathrm{b}}\left(t_{1}\right) G_{\mathrm{a}}\left(t_{1}+\tau\right) G_{\mathrm{b}}\left(t_{1}+\tau\right)+ \\
& \quad+v^{2} \varepsilon^{2} W^{2} \int \mathrm{d} t_{1} G_{\mathrm{a}}\left(t_{1}\right) G_{\mathrm{a}}\left(t_{1}+\tau\right) \int \mathrm{d} t_{2} G_{\mathrm{b}}\left(t_{2}\right) G_{\mathrm{b}}\left(t_{2}+\tau\right) .
\end{aligned}
$$

The first term is just $\left\langle I_{\mathrm{c}}\right\rangle^{2}$ and only contributes to the noise spectrum at $\omega=0$. The second term is smaller than the remaining two for small $\varepsilon$ and can be dropped; it is due to a pair of true coincidences. The third and fourth terms can be identified as the fluctuations due to chance and true coincidences. If the mixer output is integrated for time $T$ (which can be identified with our averaging period), the relative error in the charge

$$
\begin{aligned}
Q_{\mathrm{c}}= & \int_{0}^{T} \mathrm{~d} t I_{\mathrm{c}}(t) \text { is } \\
& \Delta Q_{\mathrm{c}} /\left\langle_{\mathrm{c}} Q\right\rangle=\sqrt{ }\left\{(1+2 v R / W) / v \varepsilon^{2} W T\right\}
\end{aligned}
$$

where the resolving time $R$ is given by

$R=\frac{1 \int \mathrm{d} t_{1} G_{\mathrm{a}}\left(t_{1}\right) \int \mathrm{d} t_{2} G_{\mathrm{b}}\left(t_{2}\right) \int \mathrm{d} \tau G_{\mathrm{a}}\left(t_{1}+\tau\right) G_{\mathrm{b}}\left(t_{2}+\tau\right)}{\left.2 \int \mathrm{d} t_{1} G_{\mathrm{a}}\left(t_{1}\right) G_{\mathrm{b}}\left(t_{1}\right)\right]^{2}}$.

\subsection{FourIER REPRESENTATION}

This last result, and many others, can be understood better in terms of the frequency dependence than the time dependence of the currents ${ }^{5}$ ). Our model can be rewritten by systematic use of the Fourier transform

$$
\begin{aligned}
\tilde{I}(\omega) & =\int_{-\infty}^{\infty} \mathrm{d} t I(t) \exp (+\mathrm{i} \omega t), \\
I(t) & =\int_{-\infty}^{\infty}(\mathrm{d} \omega / 2 \pi) \tilde{I}(\omega) \exp (-\mathrm{i} \omega t) .
\end{aligned}
$$

From eq. (3') we obtain

$$
\langle\tilde{I}(\omega)\rangle=2 \pi \delta(\omega) v \varepsilon \tilde{F}(0),
$$

and from eq. $\left(6^{\prime}\right)$

$$
\left\langle\tilde{I}\left(\omega^{\prime}\right) \tilde{I}(\omega)\right\rangle=2 \pi \delta\left(\omega^{\prime}+\omega\right) S(\omega),
$$

where $S(\omega)$ is called the power spectrum,

$$
\begin{aligned}
S(\omega)=\int_{-\infty}^{\infty} \mathrm{d} \tau & \exp (\mathrm{i} \omega \tau)\langle I(0) I(\tau)\rangle= \\
& =2 \pi \delta(\omega) \nu^{2} \varepsilon^{2}|\tilde{F}(0)|^{2}+v \varepsilon|\tilde{F}(\omega)|^{2} .
\end{aligned}
$$

These results show that our model of a single counter is equivalent to a source of currents $I(\omega)$ with random phase and with mean intensities having the same frequency spectrum as the pulses themselves, as well as a dc component.

Similarly, the output of the mixer in fig. 2 satisfies

$\left\langle\tilde{I}_{\mathrm{c}}(\omega)\right\rangle=2 \pi \delta(\omega) v \varepsilon^{2} W \int(1 / 2 \pi) \mathrm{d} \omega_{1} G_{\mathrm{a}}\left(\omega_{1}\right) G_{\mathrm{b}}^{*}\left(\omega_{1}\right)$,

and

with

$$
\left\langle\tilde{I}_{\mathrm{c}}\left(\omega^{\prime}\right) \tilde{I}_{\mathrm{c}}(\omega)\right\rangle=2 \pi \delta\left(\omega+\omega^{\prime}\right) S_{\mathrm{c}}(\omega)
$$




$$
\begin{aligned}
S_{\mathrm{c}}(\omega)= & 2 \pi \delta(\omega)\left|v \varepsilon^{2} W \int(1 / 2 \pi) \mathrm{d} \omega_{1} \widetilde{G}_{\mathrm{a}}\left(\omega_{1}\right) \tilde{G}_{\mathrm{b}}^{*}\left(\omega_{1}\right)\right|^{2}+ \\
& +v^{2} \varepsilon^{2} \int(1 / 2 \pi) \mathrm{d} \omega_{1}\left|\widetilde{G}_{\mathrm{a}}\left(\omega_{1}\right)\right|^{2}\left|\widetilde{G}_{\mathrm{b}}\left(\omega_{1}+\omega\right)\right|^{2}+ \\
& +v \varepsilon^{2} W\left|\int(1 / 2 \pi) \mathrm{d} \omega_{1} \widetilde{G}_{\mathrm{a}}\left(\omega_{1}\right) \tilde{G}_{\mathrm{b}}^{*}\left(\omega_{1}-\omega\right)\right|^{2}
\end{aligned}
$$

We can view the correlation apparatus as a current source with random phases and with frequency spectrum given by eq. (46). The first term in (46) gives the dc signal and the remaining terms the noise. By transforming eq. (40) [or by inspection of eq. (46)] we see that the resolving time can be expressed in terms of the pulse shapes as

$$
R=\pi \frac{\int \mathrm{d} \omega\left|\tilde{G}_{\mathrm{a}}(\omega)\right|^{2}\left|\tilde{G}_{\mathrm{b}}(\omega)\right|^{2}}{\left|\int \mathrm{d} \omega \tilde{G}_{\mathrm{a}}(\omega) \tilde{G}_{\mathrm{b}}^{*}(\omega)\right|^{2}} .
$$

This shows that the resolving time can be reduced by increasing the bandwidth of both pulses; $R$ is roughly just the reciprocal of the bandwidth of the mixer, or of the counter, whichever is smaller.

We can also generalize the discussion of the "counting time" $T$ by considering a linear filter in the mixer output. A brief derivation reproduces eq. (39) with $T$ given by

$$
T=\left[\int \frac{\mathrm{d} \omega}{2 \pi}\left|\frac{Z(\omega)}{Z(0)}\right|^{2}\right]^{-1},
$$

where $Z(\omega)$ is the transmission of the filter at each frequency. Hence $T$ is roughly the reciprocal bandwidth of the filter and meter.

At this point we feel that we have adequately proven the claims of section 2 and have shown how the model works. In the next section we will discuss (without proofs) some important extensions of this model.

\section{Extensions of the model}

The model presented in sections 2 and 3 was based on several unrealistic assumptions, introduced for clarity and simplicity: all pulses were identical, coincidences were prompt, counter a did not respond to radiation $b$, etc. These assumptions served to reduce the number of variables in the model. However, once the averaging techniques are established, it is easy to include more variables and to make the model more realistic.

\subsection{REALISTIC MODEL OF COUNTER}

The output of a real counter is influenced by more random variables than we have admitted, and will be noisier than our estimates. For example, the individual pulse heights are not identical, but are randomly distributed over a pulse height spectrum. This can be described by writing the $j^{\text {th }}$ pulse as $a_{j} F\left(t-t_{j}\right)$, where $a_{j}$ is a random amplitude. Similarly we could describe variations in shape and in arrival time. We can also include pulses in counter a due to radiation $b$; the current might then be written

$$
I_{\mathrm{a}}(t)=\sum\left[a_{j} S\left(\hat{n}^{\mathrm{a}}, \hat{n}_{j}^{\mathrm{a}}\right) F_{\mathrm{a}}\left(t-t_{j}\right)+b_{j} S\left(\hat{n}^{\mathrm{a}}, \hat{n}_{j}^{\mathrm{b}}\right) F_{\mathrm{b}}\left(t-t_{j}\right)\right]
$$

Clearly the mean current will involve $\langle a\rangle$ and $\langle b\rangle$, and the fluctuations will depend on $\left\langle a^{2}\right\rangle,\langle a b\rangle,\left\langle b^{2}\right\rangle$. Full analysis of these effects would lead to a better understanding of the influence of the counter design on the signal and the noise. For example, if we include only the effects of random amplitudes, the current fluctuation in a single counter becomes

$$
\Delta I /\langle I\rangle=\sqrt{ }\left\{\left\langle a^{2}\right\rangle /\left(v \varepsilon \Delta t\langle a\rangle^{2}\right)\right\},
$$

instead of eq. (8). This is minimized by reducing the variation between pulse heights, and (for given current) increasing the number of pulses.

\subsection{DELAYED COINCIDENCES - TIME SPECTRUM}

The extension to delayed coincidences and the measurement of perturbed angular correlations, is of considerable importance. We can accomplish this by adding another random variable for each pulse in the source, the delay time $\tau_{j}$. The two radiations $a$ and $b$ occur at times $t_{j}$ and $t_{j}+\tau_{j}$ with probability $P\left(\hat{n}_{j}^{a} \hat{n}_{j}^{\mathrm{b}} \tau_{j}\right)=$ $(\Gamma / T) \exp \left(-\Gamma \tau_{j}\right), p\left(\hat{n}_{j}^{\mathrm{a}} \hat{n}_{j}^{\mathrm{b}} \tau_{j}\right)$. Here $\Gamma^{-1}$ is the mean lifetime of the intermediate state. The cross-correlation of the two detector current becomes

$$
\begin{aligned}
& \left\langle i_{\mathrm{a}}(t) i_{\mathrm{b}}(t+\tau)\right\rangle= \\
& =v \varepsilon^{2} \int \mathrm{d} t_{1} \int \mathrm{d} \tau_{1} \Gamma \exp \left(-\Gamma \tau_{1}\right) G_{\mathrm{a}}\left(t_{1}\right) G_{\mathrm{b}}\left(t_{1}+\tau-\tau_{1}\right) W\left(\tau_{1}\right),
\end{aligned}
$$

where $W(\tau)$ is the perturbed correlation function. Note that as $\Gamma \rightarrow \infty$, eq. (50) approaches the previous result, eq. (37). The integral in eq. (50) is just $\exp (-\Gamma \tau) W(\tau)$ averaged over a time interval of order $\Delta t$. Thus, the

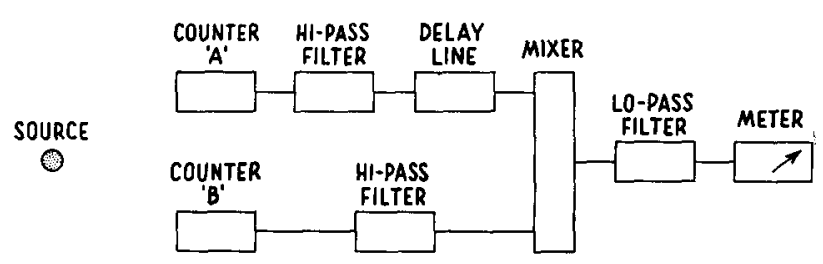

Fig. 3. Block diagram of angular correlation apparatus for measuring time spectrum of delayed coincidences. 
dc output $\left\langle I_{\mathrm{c}}\right\rangle=\left\langle i_{\mathrm{a}}(t) i_{\mathrm{b}}(t+\tau)\right\rangle$ of the mixer in fig. 3 measures the time spectrum of delayed coincidences, with finite time resolution of order $\Delta t$. To predict the fluctuations in the output of this circuit we must evaluate $\left\langle I_{\mathrm{c}}(t) I_{\mathrm{c}}\left(t^{\prime}\right)\right\rangle=\left\langle i_{\mathrm{a}}(t) i_{\mathrm{b}}(t+\tau) i_{\mathrm{a}}\left(t^{\prime}\right) i_{\mathrm{b}}\left(t+\tau^{\prime}\right)\right\rangle$. This can be shown to lead once again to eq. (39) with resolving time given by

$$
\begin{aligned}
R=\frac{1}{2 \int(\tau) \int \mathrm{d} t_{1} G^{\mathrm{a}}\left(t_{1}\right) \int \mathrm{d} t_{2} G^{\mathrm{b}}\left(t_{2}\right)} \\
\cdot \frac{\int \mathrm{d} \tau^{\prime} G^{\mathrm{a}}\left(t_{1}+\tau^{\prime}\right) G^{\mathrm{b}}\left(t_{2}+\tau^{\prime}\right)}{\left[\int \mathrm{d} t_{1} G^{\mathrm{a}}\left(t_{1}\right) G^{\mathrm{b}}\left(t_{1}+\tau-\tau_{1}\right)\right]^{2}}
\end{aligned}
$$

This shows that the resolving time is about the pulse width, but is further reduced by a factor $\exp (-\Gamma \tau)$ for long delay times. These results are a natural extension of the prompt coincidences.

\subsection{DELAYED COINCIDENCES - FREQUENCY SPECTRUM}

Next we will design a circuit to measure directly the frequency spectrum of $W(\tau)$, instead of the time spectrum. Instead of multiplying the counter outputs we will add them, select the desired frequency and then square the result; see fig. 4 . The current input to the narrow band filter is $I(t)=i_{\mathrm{a}}(t)+i_{\mathrm{b}}(t)$. The autocorrelation of this current is

$$
\begin{aligned}
& \langle I(t) I(t+\tau)\rangle= \\
& =v \varepsilon_{\mathrm{a}} \int \mathrm{d} t_{1} G^{\mathrm{a}}\left(t_{1}\right) G^{\mathrm{a}}\left(t_{1}+\tau\right)+v \varepsilon_{\mathrm{b}} \int \mathrm{d} t_{1} G^{\mathrm{b}}\left(t_{1}\right) G^{\mathrm{b}}\left(t_{1}+\tau\right)+ \\
& \quad+v \varepsilon^{2} \int \mathrm{d} t_{1} \int \Gamma \mathrm{d} \tau_{1} \exp \left(-\Gamma \tau_{1}\right) W\left(\tau_{1}\right) \\
& \quad \cdot\left\{G^{\mathrm{a}}\left(t_{1}\right) G^{\mathrm{b}}\left(t_{1}+\tau-\tau_{1}\right)+G^{\mathrm{a}}\left(t_{1}+\tau\right) G^{\mathrm{b}}\left(t_{1}-\tau_{1}\right)\right\}
\end{aligned}
$$

The first two terms are due to the auto-correlation of individual pulses, and extend over times of order $\Delta t$.

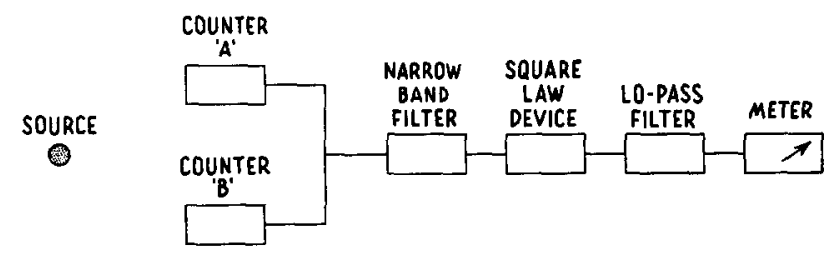

Fig. 4. Block diagram of angular correlation apparatus for measuring frequency spectrum of delayed coincidences. Note that there is no delay line in the circuit.
The last term is due to true delayed coincidences and extends over times of order $\Gamma^{-1}$.

To analyze the filter output, it is convenient to change to the frequency spectrum of this result. We find $\langle\tilde{I}(\omega)\rangle$ is zero, and

$$
\left\langle\tilde{I}\left(\omega^{\prime}\right) \tilde{I}(\omega)\right\rangle=2 \pi \delta\left(\omega+\omega^{\prime}\right) S(\omega)
$$

where the power spectrum is now

$$
\begin{aligned}
S(\omega)= & v \varepsilon_{\mathrm{a}}\left|\widetilde{G}^{\mathrm{a}}(\omega)\right|^{2}+v \varepsilon_{\mathrm{b}}\left|G^{\mathrm{b}}(\omega)\right|^{2}+ \\
& +v \varepsilon^{2}\left\{\widetilde{G}^{\mathrm{a}}(\omega) \widetilde{G}^{\mathrm{b} *}(\omega) \cdot\right. \\
& \left.\cdot \int \mathrm{d} \tau \Gamma \exp (-\Gamma \tau) W(\tau) \exp (-i \omega \tau)+\text { c.c. }\right\} .
\end{aligned}
$$

There is a simple interpretation of this: the random coincidences in each counter provide a current with broad spectrum and random phases; the true delayed coincidences give an additional contribution at the frequencies of $W(\tau)$, with frequency width $\Gamma^{-1}$ and with random phase. Neither of these gives a net average current, but both contribute to the power.

The output of the filter can be written

$$
J(t)=\int \mathrm{d} t^{\prime} H\left(t-t^{\prime}\right) I(t)
$$

or in terms of the Fourier amplitudes

$$
\tilde{J}(\omega)=\tilde{H}(\omega) \tilde{I}(\omega),
$$

where $\tilde{H}(\omega)$ is the transmission of the filter, assumed to be zero except in a narrow band. The mean output of the square law detector is

$$
\begin{aligned}
\left\langle J^{2}(t)\right\rangle & =\int \mathrm{d} t H\left(t-t^{\prime}\right) \int \mathrm{d} t^{\prime \prime} H\left(t-t^{\prime \prime}\right)\left\langle I\left(t^{\prime}\right) I\left(t^{\prime \prime}\right)\right\rangle= \\
& =\int(1 / 2 \pi) \mathrm{d} \omega|\tilde{H}(\omega)|^{2} S(\omega) .
\end{aligned}
$$

This shows that our circuit is an elementary form of a spectrum analyzer, with an output proportional to the power spectrum $S(\omega)$ integrated over a narrow band. Thus, from eq. (52) the output contains a term proportional to

$$
\tilde{W}(\omega)=\Gamma \int \mathrm{d} \tau \exp (-\Gamma \tau) \exp (-\mathrm{i} \omega \tau) W(\tau),
$$

integrated over the band pass of the filter. This term gives a bump at each of the frequencies in $W$, with width $\Gamma$. The line shape can be either Lorentzian or the dispersion curve, depending on the relative phase of the pulses. An additional delay line in one counter will shift this phase and change the line shape. 


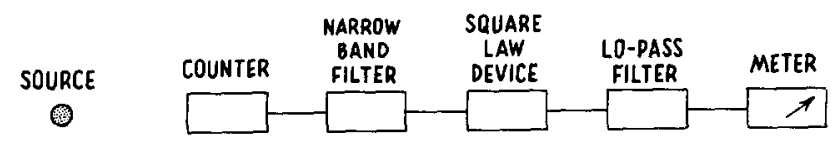

Fig. 5. Block diagram of apparatus for measuring frequency spectrum of delayed coincidences of similar radiations.

The fluctuation of this output can be evaluated by calculation of the fourth order correlation $\left\langle I(t) I\left(t^{\prime}\right) I\left(t^{\prime \prime}\right) I\left(t^{\prime \prime \prime}\right)\right\rangle$; the result is very lengthy and will not be given here. We will content ourselves with giving only the leading terms for large $v$, which can be shown to satisfy $\left\langle J^{4}(t)\right\rangle \cong 3\left\langle J^{2}(t)\right\rangle^{2}$. This implies that the noise is Gaussian, and it means that the fluctuation in the output is comparable to the mean output

$$
\sqrt{ }\left(\left\langle J^{4}\right\rangle-\left\langle J^{2}\right\rangle^{2}\right) /\left\langle J^{2}\right\rangle \cong \sqrt{ } 2
$$

If by the "signal" we mean the third term in eq. (52), then the "noise" is comparable to the first term and the signal/noise ratio is just the ratio of terms in the power spectrum, which is $\varepsilon|\tilde{W}(\omega)|$.

In this apparatus we have not succeeded in separating the true and random coincidences; both contribute to the power spectrum and give a fairly small signal/noise ratio. But there are several techniques available for improving this ratio, if the correlation is perturbed. The perturbed correlation function depends $W(\tau)$ on external fields which can be modulated, which changes the true coincidence rate but not the chance rate. Standard techniques of narrow band modulation and signal averaging should enable accurate measurement of $\tilde{W}(\omega)$.

\subsection{GAMMA-GAMMA COINCIDENCES}

Finally we note a particularly simple circuit for the angular correlation of two similar radiations, like $\gamma_{1}-\gamma_{2}$. Since one counter will respond to both radiations, we can identify the two counters in fig. 4 and simply take the output of a single counter; see fig. 5 . This circuit will measure the frequency spectrum of delayed coincidences in the same direction. The analysis of the signal and noise is the same as in section 4.3.

\section{Summary and conclusions}

We have shown the arguments to support our general thesis - that angular correlations can be measured by detecting current correlations as well as by counting pulse coincidences. The basic statistical considerations are identical: eq. (39) really sets a lower limit to the source strength, since the statistical error is independent of $v$ above a certain limit, and gets worse below this limit. Our tentative conclusion is that by raising the source strength until the current exceeds the "dark current" (which is present without any source), angular correlations can be observed in a new and different way.

Of course the principal reason for pulse counting is the good energy and time resolution which it gives. The energy resolution is lost in our circuits, which probably means that the methods discussed here are of no utility in studying complex decay schemes. In principle the energy resolution could be restored by studying the correlations in transmission through an energy sensitive device (such as a Mössbauer absorber), but this requirement is generally in conflict with the intensity requirements. The loss of energy resolution is partially offset by the frequency resolution available: a single narrow level in a complex decay scheme can be recognized by the narrow lines it produces in the apparatus of figs. 4 and 5, or by the long-lived delayed coincidences in the apparatus of fig. 3 .

Certain applications look particularly promising. One is the direct measurement of the radiofrequency spectra (Zeeman hyperfine and quadrupole splitting) of long-lived states. The apparatus in fig. 4 may be useful in bridging the gap between pulse coincidence measurements on short-lived states $(\tau \lesssim 1 \mu \mathrm{sec})$ and atomic beams measurements on long-lived states $(\tau \gtrsim 1 \mathrm{sec})$.

Another application is to precision measurements of simple decay schemes for symmetry tests. Perturbed angular correlation experiments offer an alternative to singles polarization measurements. The most interesting possibility is in measuring the frequency spectra of perturbed correlations: the "odd" terms generally have different frequencies than the "even" terms in a correlation function. For instance, the odd harmonics of the Larmor frequency in a perturbed $\beta-\gamma$ correlation are a test of parity conservation ${ }^{6}$ ); similar tests are available for perturbed $\gamma-\gamma$ correlations in quadrupole fields ${ }^{7}$ ).

\subsection{RELATION TO BROWN-TWISS INTENSITY CORRELATIONS}

The reader will note the close parallel between the circuit of fig. 2 and the stellar interferometer of Brown and Twiss ${ }^{8}$ ). Both circuits measure intensity correlations expressible in terms of a fourth order correlation of fields. We should emphasize the differences between the two devices, however: our circuit measures the correlations between two different fields (i.e. $\beta-\gamma$ or $\gamma_{1}-\gamma_{2}$ ) whereas theirs measures the correlation of one field with itself. We have tried to clarify this difference by assuming that our two counters each respond to 
only one field. The origin of the intensity correlations are due to a spatial coherence (quantum bunching) of a single field. In our apparatus, the correlations originate in the time coincidence of two different fields (or quanta) emitted by the same nucleus.

Conversations with several colleagues played a vital role in the development of this work. The author would like to acknowledge stimulating discussions with Prof. W. Williams, Dr. J. C. Van der Leeden, Dr. E. N. Kauffman and Dr. J. D. Bowman. The author is grateful to Prof. F. Boehm for the hospitality of his laboratory at Caltech, where some of this work was done.

\section{References}

1) V. M. Lobashov, V. A. Nazarenko, L. F. Saenko, L. M. Smotritskii and G. I. Kharkevich, JETP Letters 5 (1967) 59.

2) R. R. Lewis, Jr., Phys. Rev. Letters 17 (1966) 545; Phys. Rev. 163 (1967) 935.

3) S. O. Rice, Bell System Tech. J. 23 (1944) 282; 25 (1945) 46; reprinted in W. Wax, Noise and stochastic processes (Dover, 1954).

$\left.{ }^{4}\right)$ A. Davenport and B. Root, Theory of random signals and noise (McGraw-Hill, New York, 1958);

C. Lawson and D. Uhlenbeck, Threshold signals (McGrawHill, New York, 1950).

5) S. O. Rice, loc. cit., section 1.7.

$\left.{ }^{6}\right)$ L. Kruger, Z. Physik 157 (1959) 369;

F. Boehm and V. Hauser, Nucl. Phys. 14 (1959) 615.

7) R. R. Lewis, Jr., Nucl. Phys. A113 (1968) 27.

8) R. H. Brown and R. Q. Twiss, Phil. Mag. 45 (1954) 663. 